\title{
Plantas aquáticas do Parque Natural Municipal da Lagoa Comprida, Aquidauana, Mato Grosso do Sul, Brasil
}

Aquatic plants of Parque Natural Municipal da Lagoa Comprida, Aquidauana, Mato Grosso do Sul, Brazil

\author{
Lenita da Silva Vieira Ximenes', Vali Joana Pott", Camila Aokil, "II \\ Universidade Federal de Mato Grosso do Sul. Aquidauana, Mato Grosso do Sul, Brasil \\ "Universidade Federal de Mato Grosso do Sul. Campo Grande, Mato Grosso do Sul, Brasil
}

\begin{abstract}
Resumo: Este estudo teve por objetivo caracterizar a comunidade de plantas aquáticas ocorrentes no Parque Natural Municipal da Lagoa Comprida, localizado em Aquidauana, Mato Grosso do Sul. Para tanto, foram realizadas coletas mensais, entre novembro de 2012 e julho de 2013. Foram catalogadas 54 espécies, distribuídas em 27 famílias, com destaque para Cyperaceae (9 espécies), Onagraceae (5) e Poaceae (4). Sete formas de vida foram registradas, sendo as mais comuns anfíbias (24 espécies) e emergentes (19). Não foram observadas na região espécies ameaçadas de extinção ou endêmicas, contudo, considerando a extensão da lagoa, há elevada riqueza e importância para manutenção da diversidade local de hidrófitas, bem como dos organismos que dependem direta ou indiretamente destas. Há registro de espécies com potencial infestante, como Eichhornia azurea (Sw.) Kunth, Salvinia spp. e Eleocharis spp., por esta razão, melhoria no saneamento básico local e projetos de educação ambiental são prementes para manter a qualidade deste ambiente.
\end{abstract}

Palavras-chave: Florística. Hidrófitas. Lagoa urbana. Pantanal.

\begin{abstract}
The aim of this study was to characterize the community of aquatic plants that occur in the Parque Natural Municipal da Lagoa Comprida, located in Aquidauana, Mato Grosso do Sul, Brazil. Monthly collections were carried out between November 2012 and July 2013. A total of 54 species was cataloged, distributed in 27 families, especially Cyperaceae (9 species), Onagraceae (5), and Poaceae (4). Seven life forms were recorded, with amphibious (24 species) and emergent (19) being the most common. No endangered or endemic species were observed. Considering the extension of the lake, the high species richness shows that it is important for the maintenance of the local diversity of hydrophytes as well as of organisms that depend directly or indirectly on them. Species with the potential to be weeds, such as Eichhornia azurea (Sw.) Kunth, Salvinia spp. and Eleocharis spp., that been recorded, and for this reason an improvement in local basic sanitation and environmental education projects are essential to maintain the quality of this environment.
\end{abstract}

Keywords: Floristic. Hydrophytes. Urban lake. Pantanal.

XIMENES, L. S. V., V. J. POTT \& C. AOKI, 2017. Plantas aquáticas do Parque Natural Municipal da Lagoa Comprida, Aquidauana, Mato Grosso do Sul, Brasil. Boletim do Museu Paraense Emílio Goeldi. Ciências Naturais 12(2): 187-195.

Autora para correspondência: Camila Aoki. Universidade Federal de Mato Grosso do Sul. Campus Universitário de Aquidauana. Avenida Oscar Trindade de Barros, 740, Unidade II - Serraria. Aquidauana, MS, Brasil. CEP 79200-000 (aokicamila@yahoo.com.br).

Recebido em 23/05/2017

Aprovado em 22/08/2017

Responsabilidade editorial: Fernando da Silva Carvalho Filho

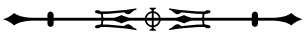




\section{INTRODUÇÃO}

O Pantanal é o maior complexo de áreas úmidas do mundo, configurando-se como uma grande planície de sedimentação, com cerca de $138.000 \mathrm{~km}^{2}$ (Silva \& Abdon, 1998). De acordo com Signor et al. (2010, p. 13),

as diferenças locais no regime hidrológico, juntamente com as variações da topografia e do solo, promovem um mosaico de áreas raramente, permanentemente ou periodicamente alagadas, bem como áreas que permanecem livres de inundação.

Além desta diversidade de ambientes aquáticos, há também o fato de este ser ainda um ecossistema bem preservado, o que favorece, em abundância, o estabelecimento permanente de plantas aquáticas livres e/ou flutuantes (Souza et al., 2011).

São consideradas plantas aquáticas e palustres as que possuem capacidade de ter o seu sistema radicular periódica ou permanentemente submerso, podendo, assim, ocupar ambientes úmidos por pelo menos algumas épocas do ano (Amaral et al., 2008). Esta vegetação apresenta grande importância, em razão da sua capacidade de reter sedimentos em suspensão, além de nutrientes, servindo ainda como alimento e refúgio para a fauna em ambientes aquáticos (Pott, 2007). São importantes fornecedoras de matéria orgânica para a cadeia detritívora e, em ambientes tropicais, estão entre os principais responsáveis pela maior porcentagem do material orgânico que entra no ambiente aquático através dos processos de decomposição e ciclagem de nutrientes (Trindade et al., 2010).

Os primeiros estudos com plantas aquáticas no Pantanal foram realizados por Hoehne (1923, 1948), com hidrófilas ('plantas da água') e higrófilas (que crescem em áreas úmidas), sem mencionar a sub-região onde teriam sido encontradas. Os trabalhos mais completos são os de Pott \& Pott (1997, 2000a), que compilaram listagens com mais de 240 espécies de macrófitas ocorrendo em todo o Pantanal. As informações sobre estas espécies aquáticas no Pantanal encontram-se mal distribuídas geograficamente, sendo predominantes os trabalhos realizados no Mato Grosso do Sul, nas sub-regiões (Silva \& Abdon, 1998) do Paraguai (Catian et al., 2012; Cunha et al., 2012; Aoki et al., 2017), do Nabileque (Rocha et al., 2015), do Abobral (Lehn et al., 2011) e de Aquidauana (Rocha et al., 2007; Gomes \& Aoki, 2015). Desta forma, ainda são insuficientes as informações sobre plantas aquáticas no Pantanal e há várias subregiões para as quais ainda não se tem coletas sistematizadas.

Este trabalho tem como objetivo caracterizar a comunidade de plantas aquáticas do Parque Natural Municipal da Lagoa Comprida, contribuindo para o conhecimento sobre a biodiversidade de plantas aquáticas da sub-região do Pantanal de Aquidauana.

\section{MATERIAL E MÉTODOS}

O estudo foi conduzido no Parque Natural Municipal da Lagoa Comprida (PNMLC), localizado na porção central da área urbana do município de Aquidauana, nas coordenadas $20^{\circ} 27^{\prime} 44^{\prime \prime}$ S, 55 46'26" O (Figura 1). Possui área total de 74,2 ha e lâmina d'água de aproximadamente 26,87 ha de extensão, apresentando característica semilêntica, sendo utilizada como área de lazer. $O$ clima da região é do tipo Aw, de acordo com a classificação de Peel et al. (2007).

As coletas foram realizadas mensalmente, entre novembro de 2012 e julho de 2013, abrangendo estações hídricas contrastantes (seca e cheia). Para levantamento florístico qualitativo, foi utilizado o método de caminhamento (Filgueiras et al., 1994). As espécies foram identificadas com auxilio de bibliografia pertinente, manuais de identificação (Pott \& Pott, 2000a; Amaral et al., 2008) e comparação com material do Herbário da Fundação Universidade Federal de Mato Grosso do Sul (CGMS). As exsicatas foram incorporadas ao acervo deste herbário.

As formas biológicas foram classificadas conforme Irgang et al. (1984): a) anfíbias - plantas registradas em solos saturados e/ou inundáveis; b) emergentes - plantas fixas ao substrato permanentemente submerso, com órgãos vegetativos e/ou reprodutivos emersos; c) flutuantes fixas - plantas fixas ao substrato com os órgãos vegetativos e/ou 


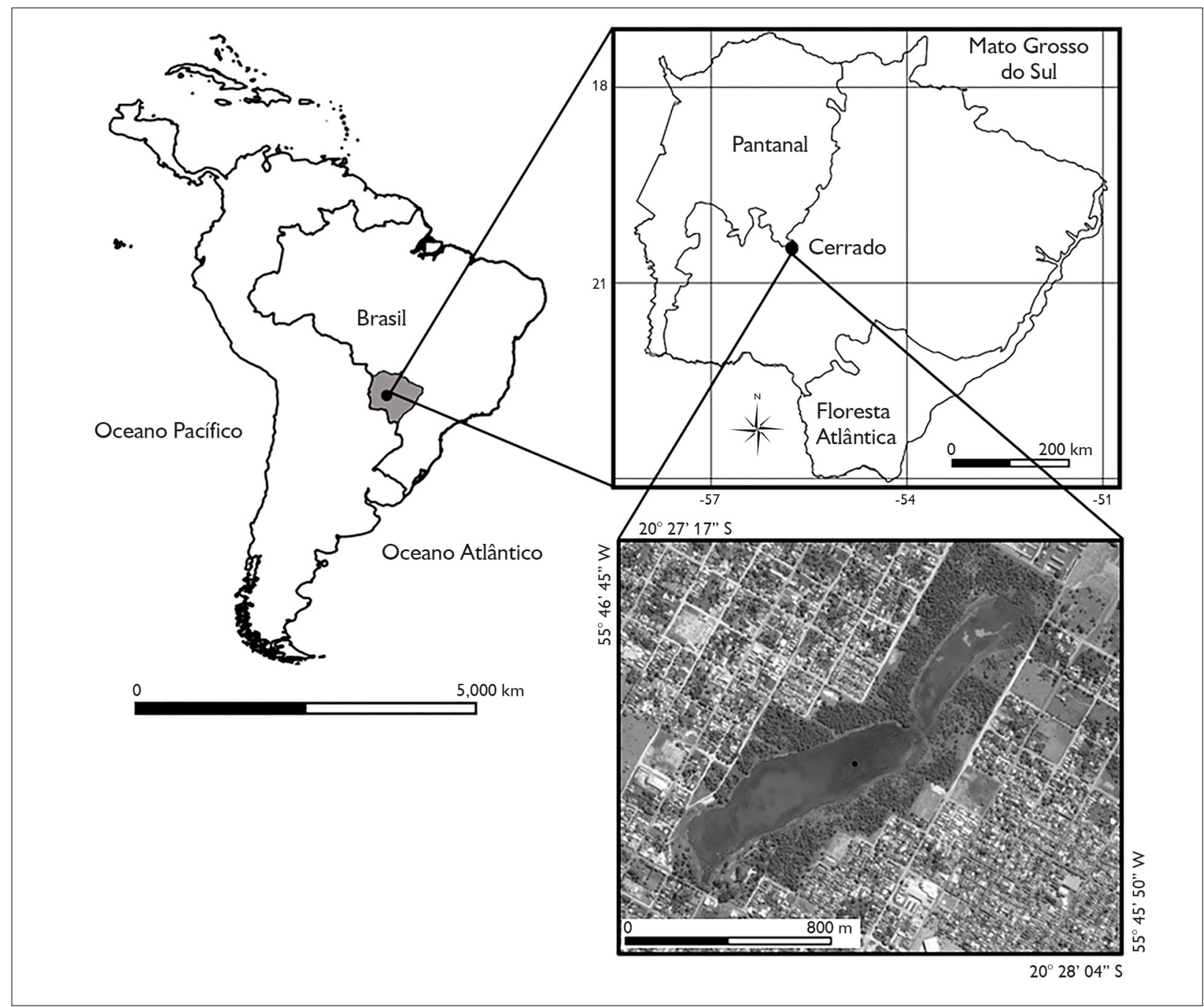

Figura 1. Localização da área de estudo no Pantanal sul-mato-grossense, sub-região de Aquidauana, Mato Grosso do Sul. Mapa confeccionado no QGis 2.2.0.

reprodutivos flutuantes; d) flutuantes livres - plantas nas quais apenas as raízes ficam imersas e os órgãos vegetativos e reprodutivos emergem; e) submersas fixas - plantas fixas ao substrato e sempre submersas; f) submersas livres plantas submersas que não permanecem fixas ao substrato; g) epífitas - espécies que crescem sobre outras plantas.

\section{RESULTADOS E DISCUSSÃO}

Foram catalogadas 54 espécies, distribuídas em 27 famílias e 41 gêneros (Apêndice, Figura 2). Das espécies registradas, apenas quatro são samambaias (Marsilea deflexa, Pityrogramma calomelanos, Salvinia auriculata e Salvinia biloba) e as demais são representantes das angiospermas. O número de espécies registradas é superior ao encontrado em lagoas no Pantanal, por Pott et al. (1989, 1992), na sub-região da Nhecolândia e de Aquidauana (37 e 49 espécies, respectivamente); é também maior do que o encontrado por Kufner et al. (2011), em lagoa meandro, na sub-região da Nhecolândia (42 espécies).

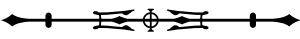



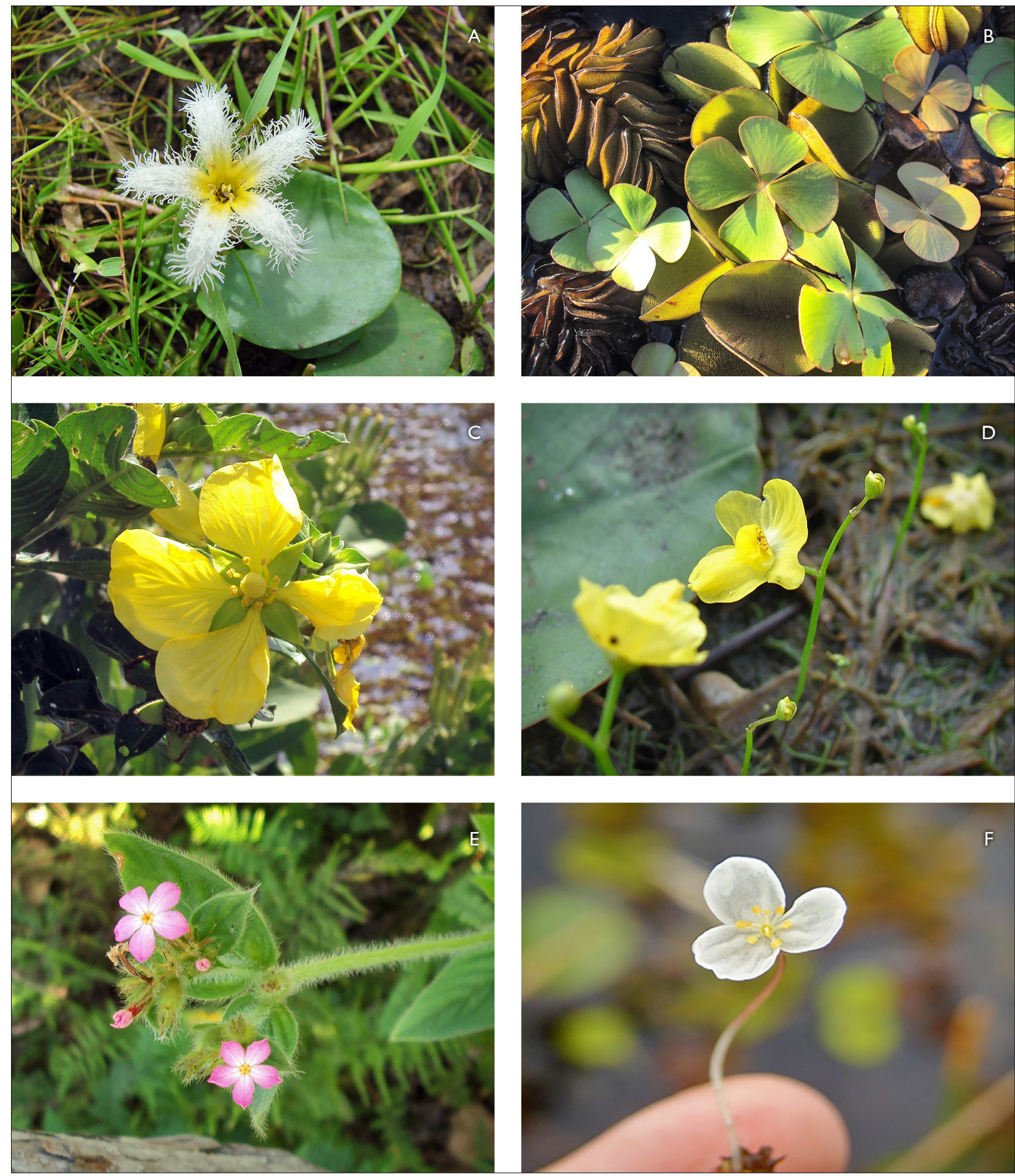

Figura 2. Espécies de plantas aquáticas registradas no Parque Natural Municipal da Lagoa Comprida, Aquidauana, Mato Grosso do Sul: A) Nymphoides indica (L.) Kuntze; B) Marsilea deflexa A. Braun; C) Ludwigia tomentosa (Cambess.) H. Hara; D) Utricularia gibba (L.); E) Sipanea hispida Benth. ex Wernahm; F) Egeria najas Planch. Fotos: Lenita Ximenes (E) e Camila Aoki. 
Contudo, a riqueza é inferior à registrada por Rocha et al. (2007) no município de Aquidauana (89 espécies), mas, neste estudo, os autores abrangeram grande variedade de corpos d'água (lagoas temporárias, permanentes e vazantes). A riqueza de espécies encontrada demonstra a importância do PNMLC para a manutenção da diversidade local de hidrófitas, bem como para os organismos que dependem direta ou indiretamente destas.

Das famílias registradas, houve destaque para Cyperaceae (9 espécies), Onagraceae (5), Poaceae (4) (Apêndice, Figura 3). De modo geral, estas famílias estão entre as mais ricas nos inventários realizados no Pantanal (Pott \& Pott, 2000b; Pott, 2007; Rocha et al., 2007). Quatorze famílias contribuíram com apenas uma espécie, entre elas Asteraceae, Pteridaceae e Rubiaceae.

Ludwigia foi o gênero mais rico (5 espécies), seguido de Cyperus e Eleocharis (com três espécies cada). Estes são, em geral, gêneros representativos em levantamentos de macrófitas aquáticas no Pantanal (Pott et al., 1992; Rocha et al., 2007). A maioria dos gêneros observados na área de estudo está representada por apenas uma espécie.

Todas as sete formas de vida propostas por Irgang et al. (1984) foram registradas na área de estudo, com predomínio de formas anfíbias (24 espécies) e emergentes (19) (Apêndice, Figura 4). Isso demonstra a elevada diversidade funcional de macrófitas aquáticas na área do PNMLC. Outros estudos no Pantanal destacaram a forma biológica emergente com sendo a mais rica (Pott et al., 1992; Cunha et al., 2012; Lehn et al., 2011). De maneira geral, os grupos ecológicos distribuem-se de maneira organizada e paralela à margem da lagoa, formando um gradiente de distribuição em direção ao interior do lago, que se inicia pelas hidrófitas anfíbias e emergentes, passando pelas espécies com folhas flutuantes até as submersas fixas (Trindade et al., 2010; Gomes \& Aoki, 2015). As espécies submersas, de modo geral, são menos frequentes, uma vez que necessitam da transparência da água para que possam realizar fotossíntese.

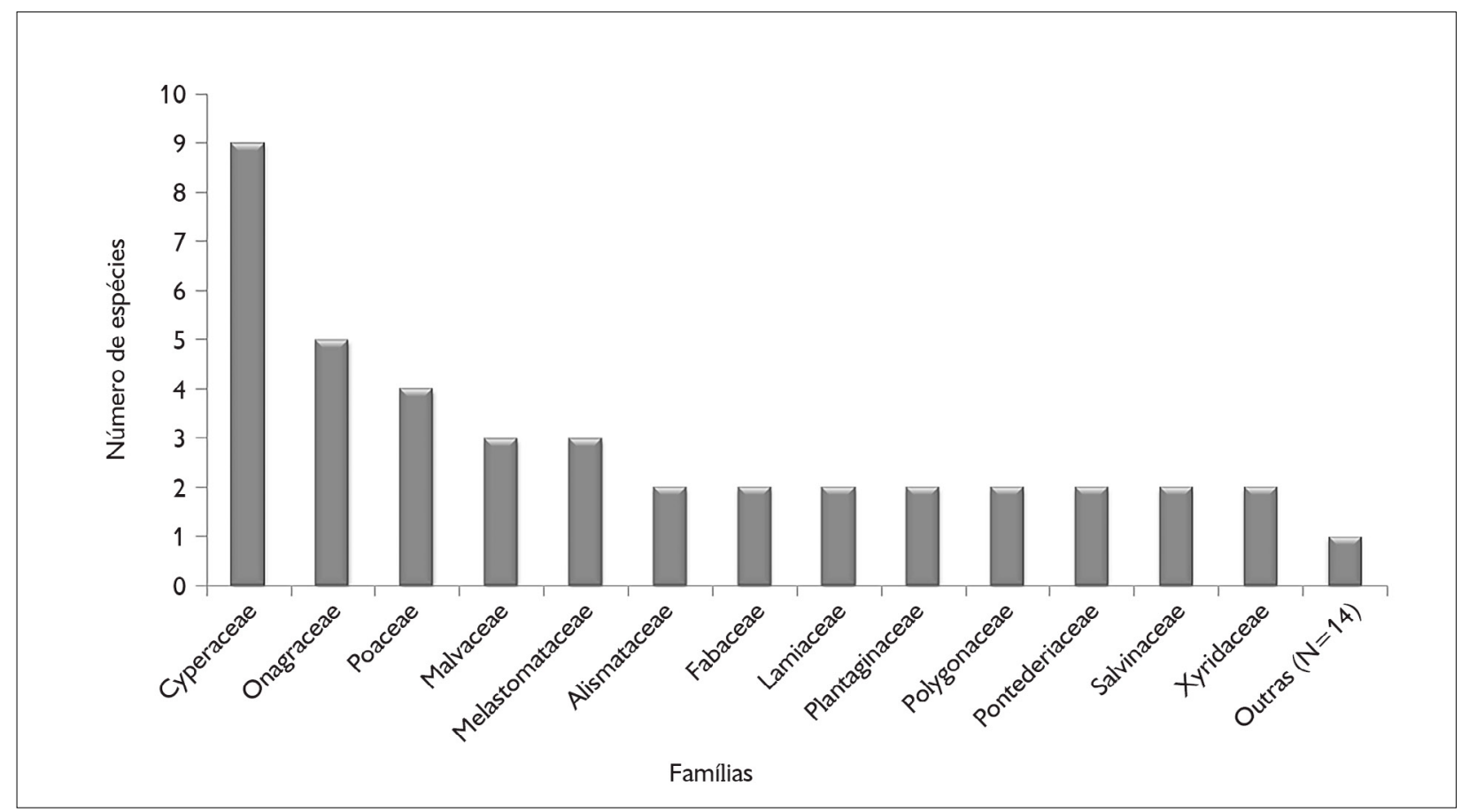

Figura 3. Contribuição de cada família com a riqueza de espécies de plantas aquáticas no Parque Natural Municipal da Lagoa Comprida, Aquidauana, Mato Grosso do Sul.

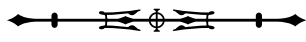




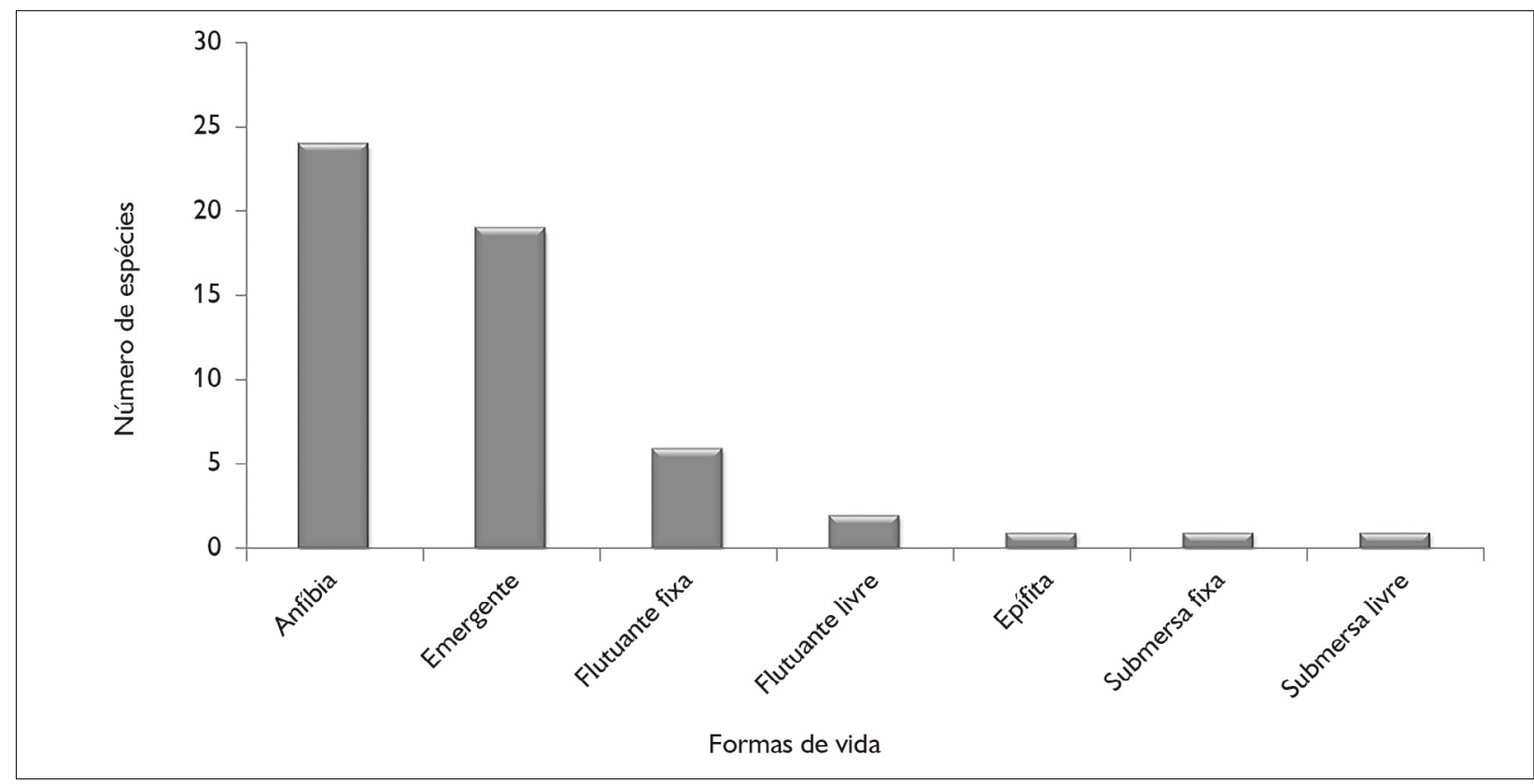

Figura 4. Formas de vida das plantas aquáticas registradas no Parque Natural Municipal da Lagoa Comprida, Aquidauana, Mato Grosso do Sul.

Não foram observadas espécies ameaçadas de extinção ou endêmicas da região, contudo, há registro de exemplares com potencial infestante, como Eichhornia azurea, Salvinia spp. e Eleocharis spp. (Pedralli, 2003), por esta razão a melhoria no saneamento básico local e o desenvolvimento de projetos de educação ambiental são prementes para manter a qualidade deste ambiente. Há lançamento de esgoto na água do local deste estudo, o que favorece o aumento da contaminação por resíduos sólidos e líquidos (Sontag \& Mello, 2013). Essas atividades geram eutrofização do corpo d'água e podem levar à mortalidade de muitas espécies de hidrófitas, bem como ao aumento exacerbado de outras (Pedralli, 2003; Camargo et al., 2003), produzindo alterações importantes do ponto de vista biológico, bem como interferindo na utilização da lagoa por parte dos moradores.

\section{CONCLUSÕES}

Há ocorrência confirmada de 54 espécies de plantas aquáticas no Parque Natural Municipal da Lagoa Comprida, riqueza relativamente alta, considerando a extensão da lagoa, sendo, desta forma, uma área importante para a manutenção da diversidade local de hidrófitas deste ambiente urbano, bem como dos organismos que dependem direta ou indiretamente destas plantas.

\section{REFERÊNCIAS}

AMARAL, M. C. E., V. BITTRICH, A. P. FARIA, L. O. ANDERSON \& L. Y. S. AONA, 2008. Guia de campo para plantas aquáticas e palustres do estado de São Paulo: 1-452. Holos, Ribeirão Preto.

AOKI, C., M. TEIXEIRA-GAMARRA, M. GAMARRA, S. C. H. MEDEIROS, V. J. POTT, G. A. DAMASCENO-JÚNIOR, A. POTT \& E. SCREMIN-DIAS, 2017. Abiotic factors drive the structure of aquatic plant assemblages in riverine habitats of the Brazilian "Pantanal". Brazilian Journal of Botany 40(2): 405-415. DOI: $<$ https://doi.org/10.1007/s40415-016-0345-0>.

CAMARGO, A. F. M, M. M. PEZZATO \& G. G. HENRY-SILVA, 2003. Fatores limitantes à produção primária de macrófitas aquáticas. In: $\mathrm{S}$. M. THOMAZ \& L. M. BINI (Ed.): Ecologia e manejo de macrófitas aquáticas: 59-83. EDUEM, Maringá.

CATIAN, G., F. M. LEME, A. FRANCENER, F. S. CARVALHO, A. POTT, V. J. POTT, E. SCREMIN DIAS, G. A. DAMASCENOJÚNIOR \& V. S. GALLETTI, 2012. Macrophyte structure in lotic-lentic habitats from Brazilian Pantanal. Oecologia Australis 16(4): 782-796. 
CUNHA, N. L., M. DELATORRE, R. B. RODRIGUES, C. VIDOTTO, F. GONÇALVES, E. SCREMIN-DIAS, G. DAMASCENO-JÚNIOR, V. J. POTT \& A. POTT, 2012. Structure of aquatic vegetation of a large lake, western border of the Brazilian Pantanal. Brazilian Journal of Biology 72(3): 519-531. DOI: <http://dx.doi.org/10.1590/S151969842012000300015>.

FILGUEIRAS, T. S., A. L. BROCHADO, P. E. NOGUEIRA \& G. F. GUALA, 1994. Caminhamento: um método expedito para levantamentos florísticos qualitativos. Caderno de Geociências (12): 39:43

GOMES, A. C. \& C. AOKI, 2015. Efeito da sazonalidade hídrica sobre a fitossociologia de macrófitas aquáticas em uma lagoa no Pantanal, Brasil. Revista de Biologia Neotropical 12(1): 1-7. DOI: <https:// doi.org/10.5216/rbn.v1i1.33966>.

HOEHNE, F. C., 1923. Phytophyononomia do Estado de Matto Grosso e ligeiras notas a respeito da composição e distribuição da sua flora: 1-104. Melhoramentos, São Paulo.

HOEHNE, F. C., 1948. Plantas aquáticas: 1-167. Instituto de Botânica, São Paulo.

IRGANG, B. E., G. E. PEDRALLI \& J. L. WAETCHER, 1984 Macrófitos aquáticos da Estação Ecológica do Taim, Rio Grande do Sul, Brasil. Roessléria 6(1): 395-404.

KUFNER, D. C. L., E. S. DIAS \& A. G. CAPORAL, 2011. Composição florística e variação sazonal da biomassa de macrófitas aquáticas em lagoa de meandro do Pantanal. Rodriguésia 62(4): 803-812. DOI: <http://dx.doi.org/10.1590/S2175-78602011000400008>.

LEHN, C. R., M. L. BUENO, D. C. L. KUFNER, E. S. DIAS, J. V. POTT \& G. A. DAMASCENO-JÚNIOR, 2011. Fitossociologia de macrófitas aquáticas associadas ao Rio Miranda, Pantanal, MS, Brasil. Revista de Biologia Neotropical 8(2): 23-31. DOI: <https://doi. org/10.5216/rbn.v8i2.12004>.

PEDRALLI, G., 2003. Macrófitas aquáticas como bioindicadoras da qualidade da água: alternativas para usos múltiplos de reservatórios. In: S. M. THOMAZ \& L. M. BINI (Ed.): Ecologia e manejo de macrófitas aquáticas: 171-188. EDUEM, Maringá.

PEEL, M. C., B. L. FINLAYSON \& T. A. MCMAHON. 2007. Updated world map of the Köppen-Geiger climate classification. Hydrology and Earth System Sciences 11(5): 1633-1644. DOI: <https://doi. org/10.5194/hess-11-1633-2007>.

POTT, V. J., 2007. Plantas aquáticas do Pantanal e da Alta Bacia. Anais do Congresso de Ecologia do Brasil 8: 1-3.

POTT, V. J., N. C. BUENO, R. A. C. PEREIRA, S. M. SALIS \& N. L. VIERA, 1989. Distribuição de macrófitas aquáticas numa lagoa na Fazenda Nhumirim, Nhecolândia, Pantanal, MS. Acta Botanica Brasilica 3(2, supl. 1): 153-168. DOI: <http://dx.doi.org/10.1590/ S0102-33061989000300015>.
POTT, V. J., N. C. BUENO \& M. P. SILVA, 1992. Levantamento florístico e fitossociológico de macrófitas aquáticas em lagoas da Fazenda Leque, Pantanal, MS. Anais do Congresso da Sociedade de Botânica de São Paulo 8: 91-99.

POTT, V. J. \& A. POTT, 1997. Checklist das macrófitas aquáticas do Pantanal, Brasil. Acta Botanica Brasilica 11(2): 215-227. DOI: <http://dx.doi.org/10.1590/S0102-33061997000200010>.

POTT, V. J. \& A. POTT, 2000a. Plantas aquáticas do Pantanal: 1-404. EMBRAPA, Brasília.

POTT, V. J. \& A. POTT, 2000b. Distribuição de macrófitas aquáticas no Pantanal. Anais do Simpósio sobre Recursos Naturais e Socioeconômicos do Pantanal 3: 1-26.

ROCHA, C. G., U. M. RESENDE \& J. S. LUGNANI, 2007. Diversidade de macrófitas em ambientes aquáticos do IPPAN na Fazenda Santa Emília, Aquidauana, MS. Revista Brasileira de Biociências 5(S2): 456-458.

ROCHA, M., C. C. SANTOS JÚNIOR, G. A. DAMASCENOJÚNIOR, V. J. POTT \& A. POTT, 2015. Effect of fire on a monodominant floating mat of Cyperus giganteus Vahl in a neotropical wetland. Brazilian Journal of Biology 75(1): 114-124. DOI: <http://dx.doi.org/10.1590/1519-6984.08613>.

SIGNOR, C. A., I. M. FERNANDES \& J. PENHA, 2010. O Pantanal e o sistema de pesquisa. In: C. A. SIGNOR, I. A. FERNANDES \& J. PENHA (Org.): Biodiversidade no Pantanal de Poconé: 13-23. Centro de Pesquisa do Pantanal, Cuiabá.

SILVA, J. S. V. \& M. M. ABDON, 1998. Delimitação do Pantanal brasileiro e suas sub-regiões. Pesquisa Agropecuária Brasileira 33: 1703-1711.

SONTAG, R. B. \& I. S. MELLO, 2013. Diagnóstico sanitário do Parque Natural Municipal da Lagoa Comprida: subsídios para o manejo e gestão. Revista Eletrônica Gestão \& Saúde 4(1): 15301549. DOI: <http://dx.doi.org/10.18673/gs.v4i1.22989>.

SOUZA, R. C. S., L. F. VIANNA, L. A. PELLEGRIN, S. M. SALIS, M. COSTA \& I. BERGIER, 2011. Localização de áreas permanentes de vegetação aquática na planície de inundação do Rio Paraguai e adjacências. Anais do Simpósio Brasileiro de Sensoriamento Remoto 15: 2036-2043.

TRINDADE, C. R., S. A. PEREIRA, E. F. ALBERTONI \& C. P. SILVA, 2010. Caracterização e importância das macrófitas aquáticas com ênfase nos ambientes límnicos do campus Carreiros-FURG, Rio Grande, RS. Cadernos de Ecologia Aquática 5(2): 1-22. 
Apêndice. Espécies de hidrófitas registradas no Parque Natural Municipal da Lagoa Comprida, Aquidauana, Mato Grosso do Sul, com seus respectivos nomes populares e formas de vida.

\begin{tabular}{|c|c|c|c|}
\hline Família & Gênero/Espécie & Nome popular & Forma de vida \\
\hline \multirow[t]{2}{*}{ Alismataceae } & Helanthium tenellum (Mart. ex Schult. \& Schult. f.) Britton & Erva-do-pântano & Anfíbia \\
\hline & Sagittaria guayanensis Kunth & Lagartixa & Flutuante fixa \\
\hline Asteraceae & Erechtites hieraciifolius (L.) Raf. ex DC. & Erva-de-fogo & Anfíbia \\
\hline Charophyceae & Nitella sp. & Lodo & Submersa fixa \\
\hline Convolvulaceae & Ipomoea carnea Jacq. & Algodão-bravo & Anfíbia \\
\hline \multirow{9}{*}{ Cyperaceae } & Cyperus brevifolius (Rottb.) Endl. ex Hassk. & - & Anfíbia \\
\hline & Cyperus haspan L. & Cebolinha & Anfíbia \\
\hline & Cyperus surinamensis Rottb. & Capim-de-rosa & Anfíbia \\
\hline & Eleocharis acutangula (Roxb.) Schult. & Cebolinha & Emergente \\
\hline & Eleocharis filiculmis Kunth & - & Emergente \\
\hline & Eleocharis minima Kunth & Lodo & Emergente \\
\hline & Fuirena umbellata Rottb. & Capim-navalha & Emergente \\
\hline & Kyllinga odorata Vahl & - & Anfíbia \\
\hline & Oxycaryum cubense (Poepp. \& Kunth) Lye & Baceiro & Epífita \\
\hline Euphorbiaceae & Caperonia castaneifolia (L.) A.St.-Hil & Erva-de-bicho-branca & Emergente \\
\hline \multirow{2}{*}{ Fabaceae } & Aeschynomene sensitiva Sw. & Cortiça & Emergente \\
\hline & Clitoria falcata Lam. & - & Anfíbia \\
\hline Hydrocharitaceae & Egeria najas Planch. & Lodinho-branco & Flutuante livre \\
\hline Hydrophyllaceae & Hydrolea spinosa L. & Amoroso & Emergente \\
\hline \multirow[t]{2}{*}{ Lamiaceae } & Hyptis brevipes Poit. & - & Anfíbia \\
\hline & Hyptis microphylla Pohl ex Benth. & - & Anfíbia \\
\hline Lentibulariaceae & Utricularia gibba L. & Lodo & Submersa livre \\
\hline \multirow{3}{*}{ Malvaceae } & Melochia villosa (Mill.) Fawc. \& Rendle & - & Anfíbia \\
\hline & Pavonia angustifolia Benth. & Malva & Anfíbia \\
\hline & Peltaea obsita (Mart. ex Colla) Krapov. \& Cristóbal & - & Anfíbia \\
\hline Marsileaceae & Marsilea deflexa A. Braun & Trevo-de-quatro-folhas & Flutuante fixa \\
\hline \multirow{3}{*}{ Melastomataceae } & Pterolepis glomerata (Rottb.) Miq. & - & Anfíbia \\
\hline & Rhynchanthera novemnervia DC. & - & Anfíbia \\
\hline & Tibouchina gracilis (Bonpl.) Cogn. & - & Anfíbia \\
\hline Menispermaceae & Odontocarya tamoides (DC.) Miers & - & Anfíbia \\
\hline Menyanthaceae & Nymphoides indica (L.) Kuntze & Ninfeia & Flutuante fixa \\
\hline Nymphaeaceae & Nymphaea gardneriana Planch. & Camalote-da-meia-noite & Flutuante fixa \\
\hline \multirow{4}{*}{ Onagraceae } & Ludwigia decurrens Walter & Florzeiro & Emergente \\
\hline & Ludwigia lagunae (Morong) H. Hara & Erva-de-bicho & Emergente \\
\hline & Ludwigia leptocarpa (Nutt.) H. Hara & Florzeiro & Anfíbia \\
\hline & Ludwigia nervosa (Poir.) H. Hara & Lombrigueira & Emergente \\
\hline
\end{tabular}


Apêndice.

(Conclusão)

\begin{tabular}{|c|c|c|c|}
\hline Família & Gênero/Espécie & Nome popular & Forma de vida \\
\hline Onagraceae & Ludwigia tomentosa (Cambess.) H. Hara & Florzeiro & Emergente \\
\hline \multirow{2}{*}{ Plantaginaceae } & Bacopa myriophylloides (Benth.) Wettst. & Lodo & Anfíbia \\
\hline & Bacopa salzmannii (Benth.) Wettst. ex Edwall & - & Emergente \\
\hline \multirow{4}{*}{ Poaceae } & Andropogon bicornis L. & Rabo-de-burro & Anfíbia \\
\hline & $\begin{array}{c}\text { Urochloa arrecta (Hack. ex T. Durand \& Schinz) } \\
\text { Morrone \& Zuloaga }\end{array}$ & Braquiária-d'água & Emergente \\
\hline & Luziola fragilis Swallen & Pastinho-d'água & Anfíbia \\
\hline & Panicum repens $\mathrm{L}$. & - & Anfíbia \\
\hline \multirow{2}{*}{ Polygonaceae } & Polygonum acuminatum Kunth & Erva-de-bicho & Emergente \\
\hline & Polygonum punctatum Elliott & Erva-de-bicho & Emergente \\
\hline \multirow{2}{*}{ Pontederiaceae } & Eichhornia azurea (Sw.) Kunth & Camalote & Flutuante fixa \\
\hline & Pontederia parviflora Alexander & Orelha-de-veado & Emergente \\
\hline Pteridaceae & Pityrogramma calomelanos (L.) Link & Samambaia-do-brejo & Emergente \\
\hline Rubiaceae & Sipanea hispida Benth. ex Wernahm & - & Anfíbia \\
\hline \multirow{2}{*}{ Salviniaceae } & Salvinia auriculata Aubl. & Orelha-de-onça & Flutuante fixa \\
\hline & Salvinia biloba Raddi & - & Flutuante livre \\
\hline Vitaceae & Cissus erosa Rich. & - & Anfíbia \\
\hline \multirow{2}{*}{ Xyridaceae } & Xyris tenella Kunth & Botão-de-ouro & Emergente \\
\hline & Xyris jupicai Rich. & Cabeçudinho & Emergente \\
\hline
\end{tabular}


